\title{
Developmental spinal canal stenosis and somatotype
}

\author{
SIMON NIGHTINGALE \\ From the Department of Neurology, Queen Elizabeth Hospital, Birmingham, UK
}

SUMMARY The hypothesis that somatotype and cervical spine developmental canal stenosis may be associated has been investigated by anthropometry and measurement of lateral projection cervical spine radiographs. A significant association of canal size with somatotype has been found such that those with developmentally narrow canals are more likely to have relatively shorter long-bones, particularly in the upper arm, and longer trunks.

Degenerative cervical spine disease is common ${ }^{1}$ and can result in severe disability and pain. It is generally appreciated that where there is already pre-existing narrowing (that is, developmental stenosis) of the cervical spinal canal, then the clinical features may be more severe and may occur with relatively little degenerative disease..$^{2-5}$ Although the factors responsible for degenerative disease have been extensively studied, the pathogenesis of developmental spinal canal stenosis has not. One possible explanation is that canal size is related to overall skeletal size, but it is not the experience of most clinicians that the canals of large people are capacious nor that those of small people are stenotic. If not related to overall skeletal size, could canal dimensions be associated with a particular body shape or somatotype? The results of this study show this to be the case.

A possible association between somatotype and canal size is suggested from two sources. Firstly in achondroplasia, an autosomal dominant condition in which a particular somatotype is characterised by short long-bones and relatively normal size of head and trunk, the subjects also have very narrow canals mainly due to shortening of the pedicles which themselves grow in the manner of long-bones. By analogy with achondroplasia, one might expect that in those with idiopathic canal stenosis, short pedicles might be associated with short long-bones elsewhere. Indeed the possibility that lumbar canal stenosis is a forme fruste of achondroplasia has been considered. ${ }^{6}$ A second possible association of somatotype and canal size arises from anthropological studies between different racial groups. For example, among the North

\footnotetext{
Address for reprint requests: Dr S Nightingale, Department of Neurology, Queen Elizabeth Hospital, Queen Elizabeth Medical Centre, Edgbaston, Birmingham B15 2TH, UK.
}

Received 17 June 1988 and in revised form 11 January 1989. Accepted 16 February 1989
African nilotic tribes somatotype is strikingly slender with relatively long limbs compared with trunk length, whereas the mongoloid races are more stocky with relatively short limbs compared to trunk length. The observation that different racial groups tend to have canals of different sizes, ${ }^{78}$ suggests a possible genetic association between somatotype and canal size.

In the literature concerning vertebral canal stenosis there are a few comments that suggest that the possibility of such an association between somatotype and canal size has been considered. Most discount this possibility ${ }^{9-12}$ though McRae $^{13}$ has commented that "thick set individuals" tend to develop lumbar canal stenosis and Babin" states "it is notable that the AP diameter varies always with biotype, race and with the more or less important muscular strains in professional and sportive activities". However, in none of these references either those for or against a possible association, are data provided to substantiate the statements nor is a reference to previously published anthropometric studies provided.

\section{Methods}

The following measurements were obtained on 91 subjects (65 men, 26 women) using standard anthropometric techniques ${ }^{15}$ : standing and sitting heights; foot, tibia, upper arm and lower arm lengths; bicondylar femur and humerus diameters; bi-iliocristal and biacromial diameters; head and chest circumferences; weight. Lengths and diameters were obtained using a Harpenden stadiometer, heights using a Harpenden anthropometer, circumferences using a tapemeasure. These measurements were made to the nearest millimetre and weight was measured on a balance weighing machine to the nearest gram.

Cervical spine radiographs were obtained with a constant focus-image distance of two meters. Measurements were made of the AP diameter of the cervical spinal canal at the C6 vertebral level from the mid-point of the posterior surface of the vertebral body to the nearest part of the line formed by the junction of the laminae. Measurement was also made of 


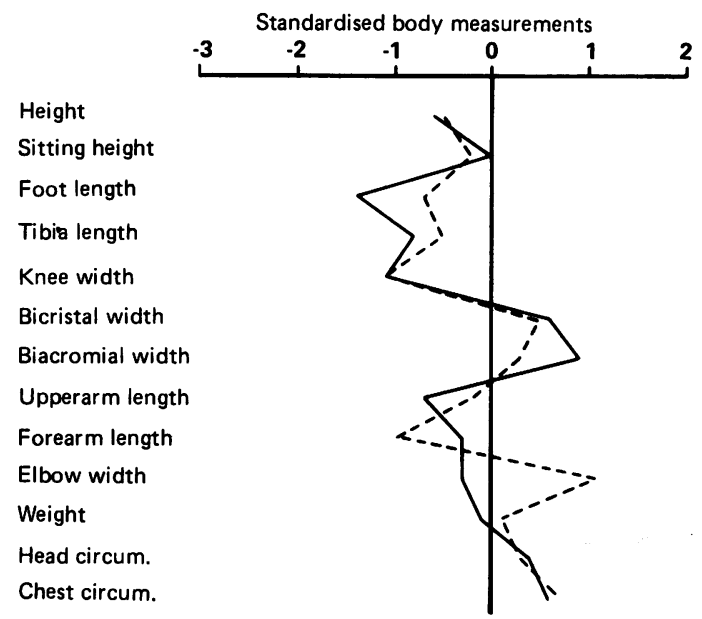

Fig 1 Pattern profiles of standardised anthropometric measurements from two patients with cervical canal stenosis to show similarity of somatotype. the image of the $5 \mathrm{~cm}$ radio-opaque marker placed sagittally over the posterior neck and a correction for magnification was made separately for each subject. The importance of such a correction has been discussed elsewhere. ${ }^{16}$

\section{Results}

To study the inter-relationships of the anthropometric variables, factor analysis was performed using "principal factoring" with an iteration procedure for improving the estimates of communalities. ${ }^{17}$ Two factors accounted for $76 \%$ of the total variance of the original data and VARIMAX rotation to a terminal solution produced a first factor that loaded mainly on "ectomorphic" variables such as height and limb length and a second factor loading mainly for "endomorphic" variables such as weight, chest circumference, etc. Knee and elbow size, head circumference and biacromial diameter loaded on both factors. A third factor with an Eigenvalue of 0.95 loaded

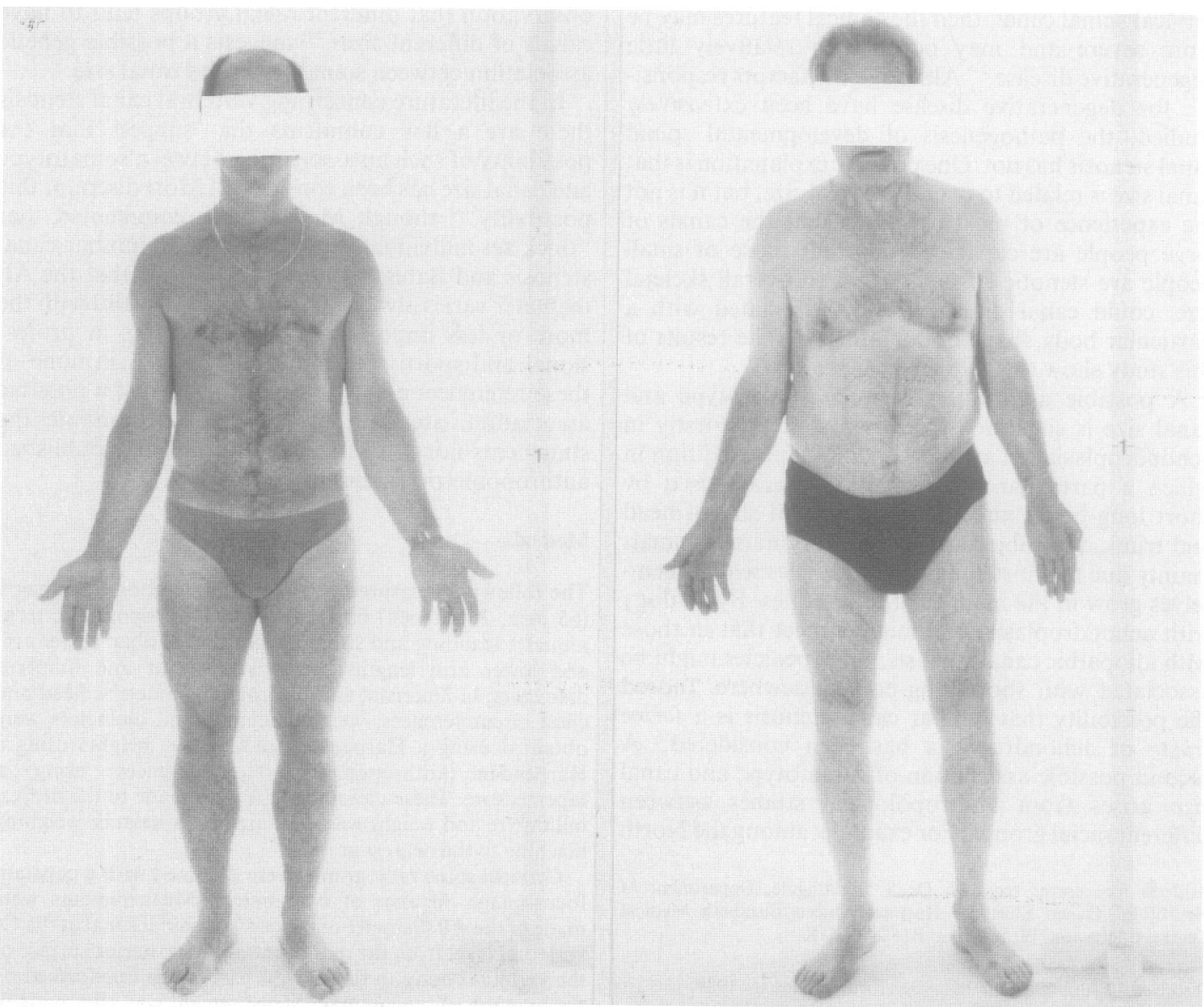

Fig 2 Two patients with cervical canal stenosis and a similar somatotype with relatively short upper arms and long trunks. 
primarily on knee width, head circumference and sitting height.

The mean antero-posterior diameter of the sixth cervical vertebral canal after correction for magnification was $14.8 \mathrm{~mm}$ (standard deviation $1.2 \mathrm{~mm}$ ) for men and $14.3 \mathrm{~mm}$ (standard deviation $1.2 \mathrm{~mm}$ ) for women.

There was a significant correlation between a number of the individual anthropometric variables and cervical canal size. The greatest correlation was between canal size and the upper arm length $(r=0.42$, $p<0.001$ ) and this was still significant after controlling for the effect of $\operatorname{sex}(r=0.37, p<0.001)$ or when males were considered alone $(r=0.38, p=0.001)$. In addition, height, the length of the foot, tibia, forearm and widths of the knee and elbow have a correlation coefficient with the canal size varying from $r=0.29$ to 0.33 ( $p<0.01$ in all instances), but controlling for the effect of sex or taking males alone largely abolished these associations. After controlling for the effect of variation in upper arm length (UAL), there remained a significant correlation of canal size with sitting height (S-H) so that "stepwise" multiple regression analysis" resulted in the following equation (all measurements in $\mathrm{mm}$ ):

Table

\begin{tabular}{lrrrrc}
\hline & $B$ & $S E B$ & $B E T A$ & $T$ & $p$ \\
\hline UAL & 0.31 & 0.06 & 0.55 & 4.8 & $<0.0001$ \\
S-H & -0.05 & 0.02 & -0.25 & -2.2 & 0.031 \\
Constant & 95.17 & 21.25 & & 4.5 & $<0.0001$ \\
\hline
\end{tabular}

In the table $B$ is the partial regression coefficient and SE B its standard error. BETA is the standardised partial regression coefficient and provides a better comparison of coefficients when the variables differ markedly in magnitude as is the case here. Pearson's correlation coefficient between the canal size as measured and that predicted by the regression model was 0.47 and $r^{2}$ was 0.22 indicating that $22 \%$ of the variation in canal size is accounted for by the regression model. The standard error of the estimate was $10.4 \mathrm{~mm}$.

Pattern profiles ${ }^{16}$ of the standardised anthropometric measurements of patients with narrow canals were superimposed to try and detect somatotypes in common (fig 1) and supported the results of the multiple regression analysis. Both of the patients in fig 2 have a somatotype of this kind and presented with symptomatic cervical canal stenosis due to a combination of developmental narrowing and degenerative factors and both required cervical decompression.

\section{Discussion}

The significant association of developmental cervical

canal narrowing and a particular somatotype has not been previously reported. The canal size tends to increase with the length of the humerus and there is a smaller independent tendency for canal size to be smaller in those with longer trunks. Although these observations are statistically significant, only $22 \%$ of the variation in developmental canal size can be related to somatotype. The factors responsible for the remaining variation in canal size may include other aspects of somatotype that can not be adequately assessed by standard anthropometry as well as inherited or acquired sources of variation unique to individual subjects. Inaccuracy in the measurement of the cervical radiographs, sitting height or limb length is unlikely to be responsible for much of the residual variation as the standard errors of these measurements are small, particularly when compared to the total range of each of these variables. ${ }^{16}$

These observations indicate that cervical developmental stenosis is part of a generalised skeletal misproportion and that the pre- and postnatal processes that determine the adult skeletal proportions affect the relationship of cervical canal size to the long bones and body length. As well as providing a new insight into the pathogenesis of developmental stenosis, the findings are of potential clinical value. Although the large standard error of the estimate prevents accurate prediction of cervical canal size from somatotype, the presence of a relatively short upper arm and long trunk in a patient with spinal symptoms may alert the physician to the possibility of developmental canal stenosis and the consequently greater risk of the expression of degenerative spine disease.

I am grateful for the help of the Departments of Neurology and Radiology of the Royal Victoria Infirmary, Newcastle upon Tyne.

Correlation matrices and summary statistics for the anthropometric variables will be provided by the author on request.

\section{References}

1 Lawrence JS. Disc degeneration. Its frequency and relationship to symptoms. Ann Rheum Dis 1969; 28:121-38.

2 Payne EE, Spillane JD. The cervical spine: an anatomicopathological study of 70 specimens (using a special technique) with particular referece to the problem of cervical spondylosis. Brain 1957;80:571-93.

3 Hinck VS, Sachdev NS. Developmental stenosis of the cervical spinal canal. Brain 1966;89:27-36.

4 Nurick S. The pathogenesis of the spinal cord disorder associated with cervical spondylosis. Brain 1972;95: 87-100.

5 Epstein BS, Epstein JA, Jones MD. Cervical spinal stenosis. Radiol Clin North Am 1977;15:215-25.

6 Roberts GM. Lumbar stenosis. MD Thesis. University of 
London, 1978.

7 Eisenstein S. Measurement of the lumbar spinal canal in two racial groups. Clin Orthop 1976;115:42-46.

8 Postacchini F, Ripani M, Carpano S. Morphometry of the lumbar vertebrae. An anatomic study of two Caucasoid ethnic groups. Clin Orthop 1983;172: 296-303.

9 Verbiest H. Neurogenic intermittent claudication. With special reference to stenosis of the lumbar vertebral canal. New York: Elsevier, 1976.

10 Verbiest $H$. Pathomorphologic aspects of developmental lumbar stenosis. Orthop Clin North Am 1975;6:177-96.

11 Verbiest, H. Stenosis of the lumbar vertebral canal and sciatica. Neurosurg Rev 1980;3:75-89.

12 Weinstein PR. The application of anatomy and pathophysiology in the management of lumbar spine disease. Clin Neurosurg 1980;27:517-40.
13 McRae DL. Radiology of the lumbar spinal canal. In: Weinstein PR, Ehni G, Wilson CB, eds. Lumbar Spondylosis: Diagnosis, Management and Surgical Treatment. London: Year Bk Med, 1977:92-114.

14 Babin E. Radiology of the narrow lumbar canal. In: Wackenheim A, Babin E, eds. The Narrow Lumbar Canal. Radiologic Signs and Surgery. New York: Springer-Verlag, 1980:1-10.

15 Cameron N. The methods of auxological anthropometry. In: Falkener F, Tanner JM, eds. Human Growth. New York: Plenum Pub, 1978:35-90.

16 Nightingale S. Developmental vertebral canal stenosis. A clinical, radiological, anthropometric and genetic study. MD Thesis. University of London, 1986.

$17 \mathrm{Kim}$ JO. Factor analysis. In: Nie NH, Hull CH, Jenkins JG, eds. SPSS Users Guide. New York: McGraw, 1983:468-514. 